\title{
Broadband transverse displacement sensing of silicon hollow nanodisk under focused radial polarization illumination in the near-infrared region
}

\author{
Jianxin Wang (王建金金) $)^{1}$, Xianghui Wang (王湘晖) $)^{1, *}$, and Ming Zeng (曾 明) ${ }^{2}$ \\ ${ }^{1}$ Institute of Modern Optics, Tianjin Key Laboratory of Micro-scale Optical Information Science and Technology, \\ Nankai University, Tianjin 300350, China \\ ${ }^{2}$ School of Electrical Engineering and Automation, Tianjin University, Tianjin 300072, China \\ *Corresponding author: wangxianghui@nankai.edu.cn \\ Received January 10, 2020; accepted March 2, 2020; posted online May 11, 2020
}

\begin{abstract}
Broadband transverse displacement sensing by exploiting the interaction of a focused radially polarized beam with a silicon hollow nanodisk is proposed. The multipolar decomposition analysis indicates that the interference between a longitudinal total electric dipole (TED) moment and a lateral magnetic dipole (MD) moment is dominant in the far-field transverse scattering in the near-infrared region. Within a broadband wavelength range with the width of $155 \mathrm{~nm}$, the longitudinal TED is almost in phase with the lateral MD, and then broadband position sensing based on the sensitivity of scattering directivity to transverse displacement can be achieved.

Keywords: directional scattering; transverse Kerker's condition; displacement sensing; hollow nanodisk; radial polarization.

doi: 10.3788/COL202018.063602.
\end{abstract}

In past decades, optical nanoantennas have become important fundamental elements in the field of modern nanophotonics due to their capability to manipulate light-matter interactions at the subwavelength scale $-\underline{[1-3]}$. For many nanophotonics applications, it is of great importance to direct far-field scattering of optical nanoantennas in a preferential direction ${ }^{[4,5]}$. Directional scattering from plasmonic $-[6,7]$, dielectric ${ }_{-[-10]}^{-1}$, and hybrid nanoantennas ${ }_{-11]}^{[1]}$ has been proposed and tuned by the manipulation of various geometric parameters including size, length, shape, and gap dimension. As compared with their metallic counterparts, all-dielectric nanoantennas with high refractive index exhibit low optical losses in the visible and near-infrared spectral ranges and then have attracted a lot of interest in recent years $-\underline{[8-10,12-18]}$. In addition, both electric modes and magnetic modes can be simultaneously excited in all-dielectric nanoantennas, which could provide more freedom to control directional scattering. By balancing the relative magnitude and phase differences between the electric dipole (ED) and magnetic dipole (MD), forward/backward scattering can be enhanced or suppressed when the Kerker's condition is fulfilled $\underline{[8,14,17,18]}$.

Recently, the excitation of longitudinal dipole modes in an optical nanoantenna can be more easily accessed via beam engineering ${ }^{[19]}$. For example, under focused radial polarization (RP) illumination, an ED mode along the propagation direction can be selectively excited because a strong longitudinal electric field is produced in the vicinity of the focus when an RP beam is tightly focused by an objective with a high numerical aperture (NA). When this longitudinal ED mode is further constructively superimposed with a transverse MD moment mode, and the transverse Kerker's condition is satisfied, a unidirectional lateral scattering can be induced ${ }^{[20-24]}$. Due to the inhomogeneous intensity distribution of electric and magnetic fields in the focal volume, the relative magnitude of the longitudinal ED and transverse MD modes can be tuned by the antenna's position. Therefore, lateral scattering is highly dependent on the antenna's position relative to the focus, and high-precision displacement sensing can be realized by detecting the variation of lateral scattering as a function of the antenna's position $\underline{[22-23,25-28]}$. It has been experimentally demonstrated that based on lateral scattering from a silicon spherical nanoparticle under cylindrical vector beam illumination, displacements down to a few angstroms can be resolved ${ }^{[23]}$. The measuring range of transverse displacement sensing can be tuned from several nanometers to a few micrometers by adjusting the outer and inner radii of a core-shell nanoparticle excited by a focused azimuthally polarized (AP) beam $\stackrel{[25]}{-}$ For many practical applications, displacement sensing over a broadband wavelength range is highly desired. As compared to the nanosphere with one geometric parameter, the hollow nanodisk has three tunable geometric parameters, and a broadband operating wavelength width is much more easily obtained. All-dielectric hollow nanodisks have been proposed to enhance forward scattering at different wavelengths $\underline{[29,30]}$ or over a broadband spectral region $\underline{31]}$. However, to the best of our knowledge, little attention has been paid to lateral scattering of hollow nanodisks excited by a focused beam.

Here, we investigate the interaction between a silicon hollow nanodisk and a focused RP beam. According to multipolar decomposition analysis, it is found that the interference between a longitudinal total ED (TED) moment and a lateral $\mathrm{MD}$ is dominant in transverse 
far-field scattering of the hollow nanodisk in the nearinfrared region. Within a broadband wavelength range, the two dipoles almost oscillate in phase and can interfere constructively along the lateral direction. As a result, broadband displacement sensing can be achieved, and the operating wavelength width reaches about $155 \mathrm{~nm}$.

In our proposed scheme, illustrated in Fig. 1, an RP beam is focused by an infinity-corrected objective. The NA of the focusing objective is 0.9 . A silicon hollow nanodisk with outer radius $R_{\text {out }}=140 \mathrm{~nm}$ and height $H=250 \mathrm{~nm}$ is chosen as a single-element antenna. The inner radius of the air hole in the center of the hollow nanodisk is $R_{\mathrm{in}}=30 \mathrm{~nm}$. The hollow nanodisk is embedded in a homogeneous surrounding medium. The surrounding medium is assumed to be air. The dielectric permittivity of silicon used here is adopted from Palik's handbook ${ }^{[32]}$.

The focused field in the focal region can be obtained analytically by virtue of the vector diffraction integral $\stackrel{[3]}{-3}$ and then imported to three-dimensional (3D) finite difference time domain (FDTD) simulation. The scattering intensity is numerically calculated by using the FDTD numerical method. Figure 2 shows the scattering spectrum of the hollow nanodisk with different lateral displacement along the $+x$ direction. The far-field scattering can be considered as a result induced by a superposition of electric and magnetic multipole moments, which is usually analyzed using the multipolar decomposition approach developed for the scattering of light from arbitrary-shaped nanoparticles $\stackrel{[34]}{ }$. The various multipole contributions can be obtained by integrating the induced polarization currents over the whole volume of the hollow nanodisk. For comparison, those results (up to the quadrupole terms) are also presented in Fig. 2 . The TED mode is the interference of ED and electric toroidal dipole (TD) moments because the far-field radiation patterns of ED and TD are identical and cannot be distinguished ${ }^{[35]}$. As shown in Fig. 2(a), when the hollow nanodisk locates at the focus, i.e., the lateral position $x=0$, there is only a resonance peak at $\lambda=835 \mathrm{~nm}$. Because a focused RP beam exhibits a null in the magnetic field in the focus while there is a strong longitudinal electric field $\stackrel{[33]}{ }$, magnetic modes are suppressed, and only electric modes are induced $\stackrel{[19]}{ }$. Therefore, this resonance is attributed to the electric response, which is also illustrated in the results from multipolar

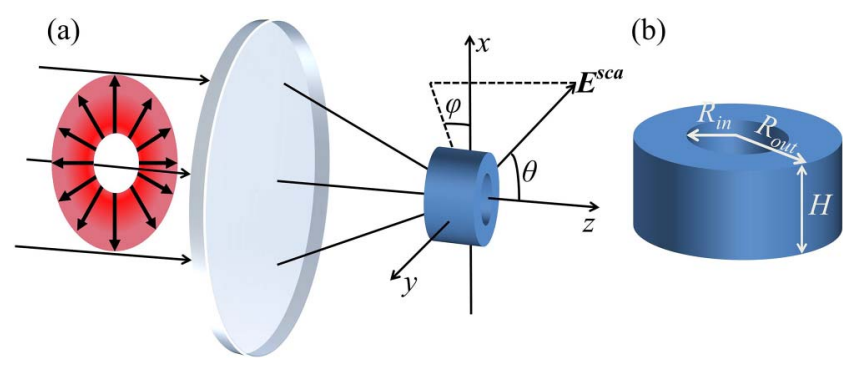

Fig. 1. (a) Schematic diagram of light scattering from a silicon hollow nanodisk illuminated by a focused RP beam. (b) Geometry of the hollow nanodisk.
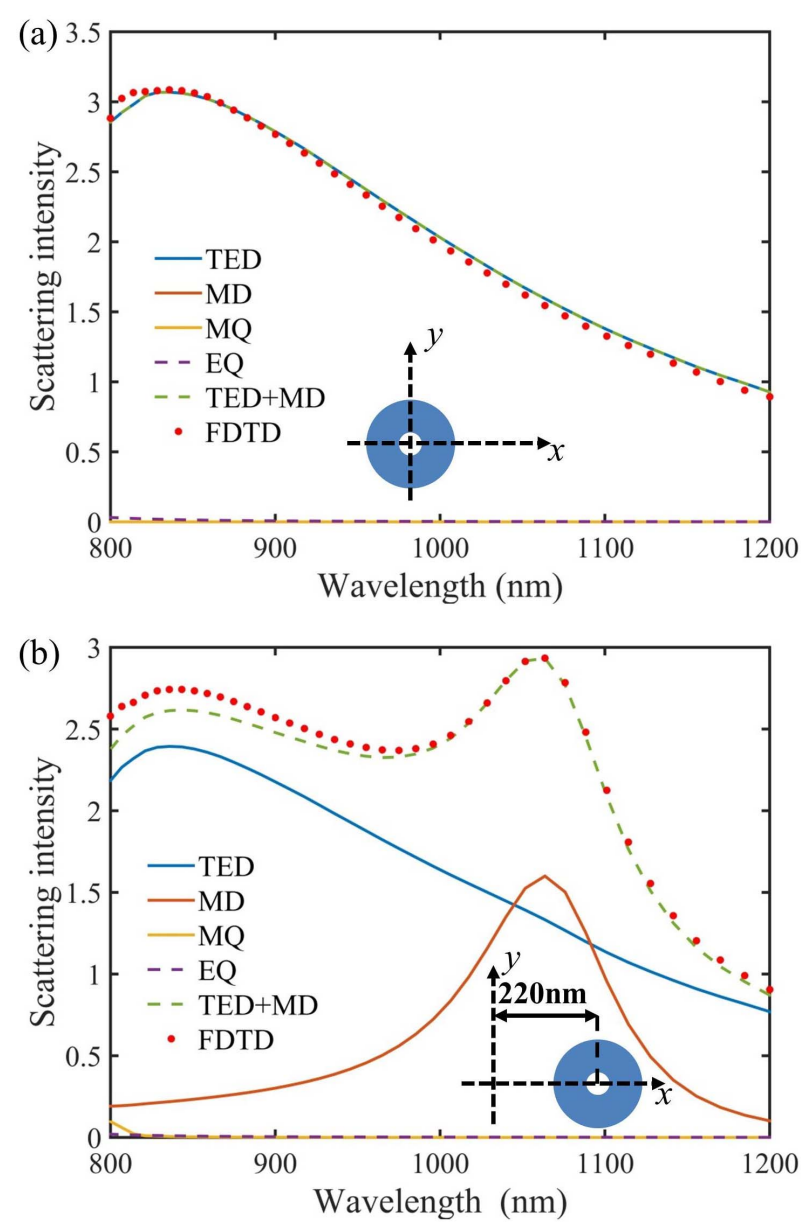

Fig. 2. Scattering spectrum of the hollow nanodisk at the position of (a) $x=0 \mathrm{~nm}$ and (b) $x=220 \mathrm{~nm}$ as a function of the incident wavelength, respectively. The green dashed lines denote the result of the overlapping of TED and MD.

decomposition analysis. The scattering spectrum of the hollow nanodisk at the position of $x=220 \mathrm{~nm}$ is given by Fig. 2(b). A new resonance peak at $\lambda=1060 \mathrm{~nm}$ occurs. Under focused RP illumination, the distribution of the magnetic field is doughnut-shaped in the focal plane ${ }^{[33]}$. As the hollow nanodisk moves away from the optical axis, the relative strength of the magnetic field increases, and then the MD response becomes stronger. According to multipolar decomposition analysis, there is a strong MD resonance. The magnetic quadrupole (MQ) contribution also increases. However, this higher-order contribution can be observed only at much shorter wavelength. The scattering intensity from the interference of TED and MD is almost in agreement with the result obtained from FDTD simulation. Therefore, it is enough to model directional scattering of the hollow nanodisk in the nearinfrared region by a superposition of TED and MD.

For a focused RP beam, the focused field presents a stronger longitudinal electric component surrounded by a much weaker lateral electric component ${ }^{[33]}$. The magnetic field is purely transversely polarized and exhibits doughnut-shaped distribution in the focal plane. When the hollow nanodisk moves away from the focus and along 
the $x$ direction, a transverse ED $p_{x}$ and a TD $t_{x}$, a longitudinal ED $p_{z}$ and a TD $t_{z}$, and a transverse MD $m_{y}$ could be excited. Following Eq. (11) in Ref. [36], the term $D$ is defined as the interference of ED and TD moments and then can be treated as TED moment. Therefore, the far-field scattering field of the hollow nanodisk can be written as

$$
\left[\begin{array}{c}
E_{\theta}^{\mathrm{sca}}(\boldsymbol{r}) \\
E_{\varphi}^{\mathrm{sca}}(\boldsymbol{r})
\end{array}\right]=\frac{k_{0}^{2} e^{i \boldsymbol{k}_{0} \cdot \boldsymbol{r}}}{4 \pi r \varepsilon_{0}}\left[\begin{array}{c}
D_{x} \cos \theta \cos \varphi-D_{z} \sin \theta+\frac{m_{y}}{c} \cos \varphi \\
-D_{x} \sin \varphi-\frac{m_{y}}{c} \cos \theta \sin \varphi
\end{array}\right],
$$

where $\boldsymbol{r}$ is an observation point in the far field. $\theta$ and $\varphi$ stand for the polar and azimuthal angles of the observed point [see Fig. 1(a)]. $\varepsilon_{0}$ and $c$ are the permittivity and speed of light in the vaccum, respectively. $\boldsymbol{k}$ is the freespace wave number. According to Eq. (1),$D_{x}$ has no contribution to far-field scattering along the $x$ direction. The unidirectional lateral scattering along the $+x$ direction could be observed when

$$
\left|m_{y}\right|=\left|c D_{z}\right|, \quad \arg \left(m_{y}\right)-\arg \left(c D_{z}\right)=180^{\circ} .
$$

Similarly, the condition for the $-x$ unidirectional scattering could be written as

$$
\left|m_{y}\right|=\left|c D_{z}\right|, \quad \arg \left(m_{y}\right)-\arg \left(c D_{z}\right)=0^{\circ} .
$$

Figure $\underline{3(\mathrm{a})}$ presents the amplitude ratio of $D_{z}$ and $m_{y}$ for the different lateral displacements of the nanoparticle's position relative to the focus along the $+x$ direction. Due to the inhomogeneous intensity distributions of the longitudinal electric field and transverse magnetic field $[21,23,33$, the relative strength of $\left|m_{y} / c D_{z}\right|$ increases as the hollow nanodisk is moved away from the optical axis. According to the dashed line, for the wavelengths of $1030 \mathrm{~nm}$, $1007 \mathrm{~nm}, 985 \mathrm{~nm}, 955 \mathrm{~nm}, 918 \mathrm{~nm}$, and $875 \mathrm{~nm}$, the ratio reaches one when the lateral displacement is $220 \mathrm{~nm}$, $250 \mathrm{~nm}, 280 \mathrm{~nm}, 315 \mathrm{~nm}, 340 \mathrm{~nm}$, and $355 \mathrm{~nm}$, respectively. As the lateral displacement continues to increase, the ratio correspondingly increases until the amplitude of $m_{y}$ reaches a maximum. Figure $\underline{3(\mathrm{~b})}$ gives phase difference of $D_{z}$ and $m_{y}$ as a function of the incident wavelength. As the lateral displacement increases from $80 \mathrm{~nm}$ to $355 \mathrm{~nm}$, there is no variation in the phase difference between the longitudinal electric field and transverse magnetic field ${ }^{[23]}$. The phase difference of $D_{z}$ and $m_{y}$ is only relative to the incident wavelength. As marked with the two horizontal dashed lines, the phase difference of those two dipole moments does not exceed $7^{\circ}$ in the spectral range of $875-1030 \mathrm{~nm}$. Therefore, $D_{z}$ is almost in phase with $m_{y}$ in this broad wavelength range with the width of $155 \mathrm{~nm}$, which implies that broadband lateral scattering along the $-x$ direction can be realized. For a nanosphere with the radius $R=140 \mathrm{~nm}$, the phase difference of $D_{z}$ and $m_{y}$ is also plotted in Fig. $\underline{3(\mathrm{~b})}$. $D_{z}$ is in phase with $m_{y}$ at two individual wavelengths, which is similar to the
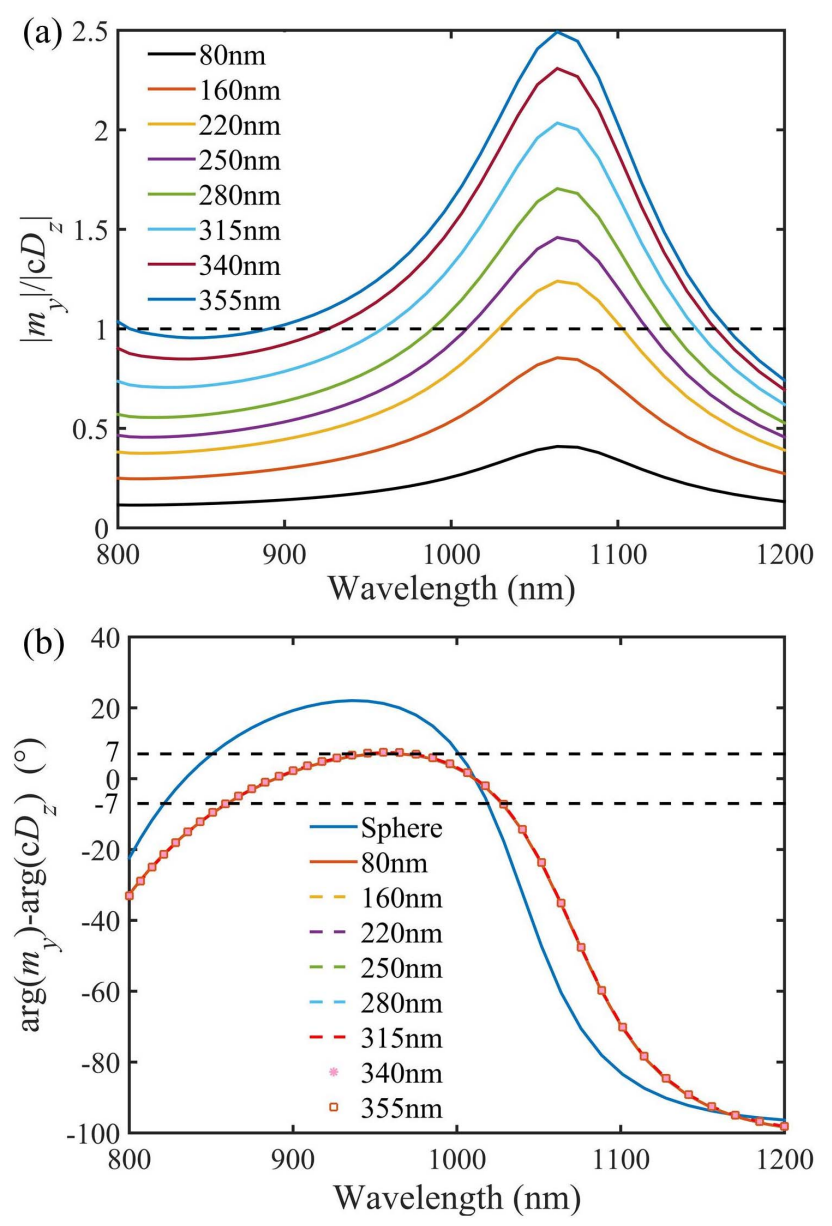

Fig. 3. (a) Relative amplitude and (b) phase difference between $D_{z}$ and $m_{y}$ for the different lateral displacements.

result in Ref. [23]. The wavelength bandwidths are about $30 \mathrm{~nm}$ and $20 \mathrm{~nm}$, respectively.

For the wavelength of $1030 \mathrm{~nm}$, the 3D far-field radiation pattern of the scattering field when the hollow nano-

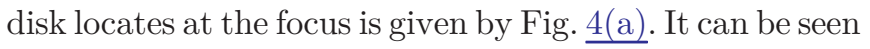
that the far-field scattering exhibits a donut-like shape induced by a longitudinal $D_{z}$. The polar plot in Fig. 4(b) illustrates that there are two symmetric lobes in the $x z$ plane along the $x$ direction. According to the result in Fig. $\underline{4(\mathrm{c})}$, when the hollow nanodisk is positioned at $x=220 \mathrm{~nm}$, far-field directional scattering dominantly propagates along the $-x$ direction, and a unidirectional lateral scattering is observed. Figure $\underline{4(d)}$ gives polar plots of directional scattering for different wavelengths. For the wavelengths of $1007 \mathrm{~nm}, 985 \mathrm{~nm}, 955 \mathrm{~nm}, 918 \mathrm{~nm}$, and $875 \mathrm{~nm}$, the magnitude of lateral magnetic response is comparable to that of the longitudinal electric response, and leftward unidirectional scattering can also be induced when the lateral displacement is $250 \mathrm{~nm}, 280 \mathrm{~nm}, 315 \mathrm{~nm}$, $340 \mathrm{~nm}$, and $355 \mathrm{~nm}$, respectively. The far-field scattering along the exact $z$ direction occurs, especially for shorter wavelengths, which is attributed to the influence from $D_{x}$. Because the focused radially polarized field is rotationally symmetrical about the optical axis, the same variation in the radiation pattern presented in Fig. $\underline{4}$ could be observed 

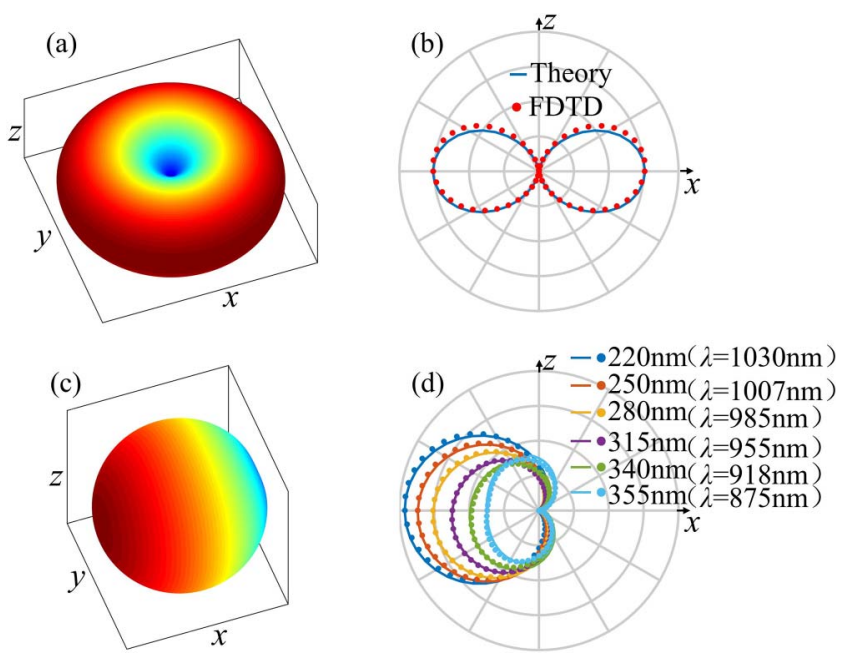

Fig. 4. (a) 3D radiation pattern and (b) polar plot of the scattering field for the wavelength of $1030 \mathrm{~nm}$ when $x=0 \mathrm{~nm}$. (c) 3D radiation pattern for the wavelength of $1030 \mathrm{~nm}$ when $x=220 \mathrm{~nm}$. (d) Polar plots of the scattering field for different wavelengths when $x=220 \mathrm{~nm}, 250 \mathrm{~nm}, 280 \mathrm{~nm}, 315 \mathrm{~nm}, 340 \mathrm{~nm}$, and $355 \mathrm{~nm}$, respectively. The solid dots and lines in (b) and (d) give the results obtained by FDTD simulation and the theoretical model, including the overlapping of TED and MD, respectively.

as the hollow nanodisk moves along the other radial direction, for example, the $y$ axis.

In order to distinctly illustrate the dependence of lateral scattering on the nanoparticle's position over a broad wavelength range, polar plots of directional scattering in the $x z$ plane as a function of the lateral displacement at the wavelength of $1030 \mathrm{~nm}$ and $875 \mathrm{~nm}$ are presented by Figs. $\underline{5(\mathrm{a})}$ and $5(\mathrm{~b})$, respectively. As shown in Fig. $\underline{5(\mathrm{a})}$, for the wavelength of $1030 \mathrm{~nm}$, with the increasing lateral displacement of the hollow nanodisk, one of the lobes along the $+x$ direction begins to shrink, and the far-field scattering pattern progressively changes from a symmetric two-lobe structure to an asymmetric radiation pattern. The cases for other wavelengths are similar. In one word, the lateral scattering directivity is highly positionsensitive over a broad wavelength range. Conversely, the variation of the scattering directivity can be utilized to measure the lateral position of the hollow nanodisk relative to the optical axis.

The lateral scattering directivity can be quantitatively investigated by the left-to-right ratio (LRR) in decibels $(\mathrm{dB})$, which is defined as

$$
\mathrm{LRR}=10 \log _{10}\left[\frac{E_{-x}^{2}\left(\varphi=180^{\circ}, \theta=90^{\circ}\right)}{E_{+x}^{2}\left(\varphi=0^{\circ}, \theta=90^{\circ}\right)}\right] .
$$

According to Eq. (1), it can be rewritten as

$$
\mathrm{LRR}=20 \log _{10}\left[\frac{1+m_{y} /\left(c D_{z}\right)}{1-m_{y} /\left(c D_{z}\right)}\right] .
$$
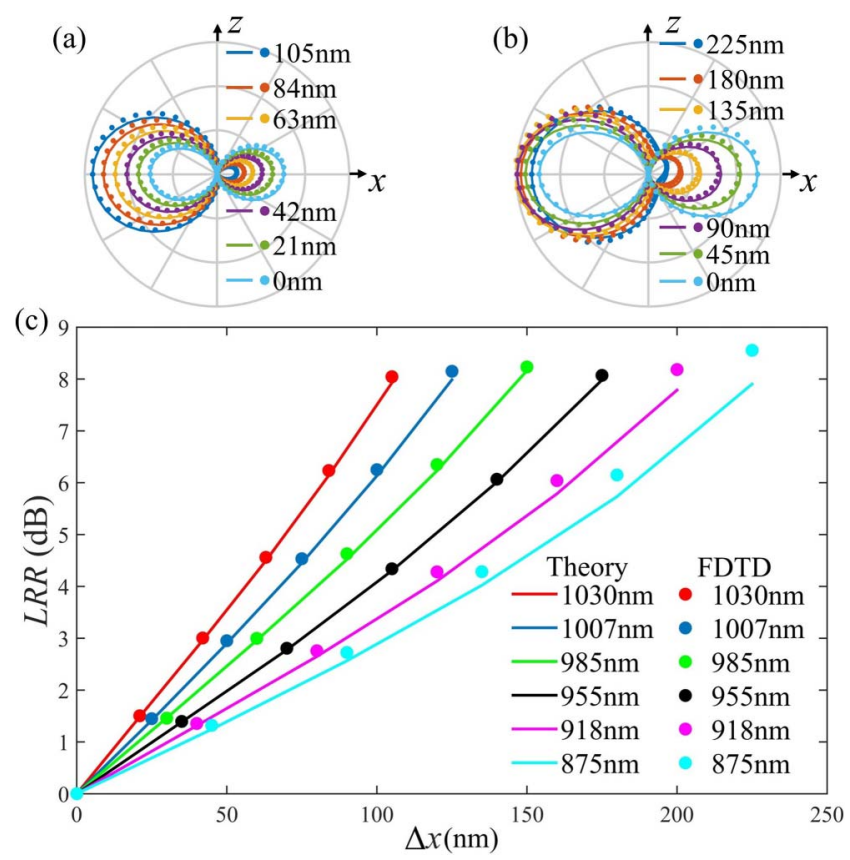

Fig. 5. Far-field scattering for different lateral displacements at the wavelength of (a) $1030 \mathrm{~nm}$ and (b) $875 \mathrm{~nm}$ in the $x z$ plane, respectively. (c) The variations of lateral directivity as a function of lateral displacement for different wavelengths.

The LRR is dependent on the relative ratio of $m_{y} / c D_{z}$, which is relative to the antenna's position in the focal volume.

The variations of lateral directivity as a function of transverse displacement $\Delta x$ for different wavelengths are given by Fig. $5(\mathrm{c})$. At the wavelength of $1030 \mathrm{~nm}$, the scattering directivity increases approximately linearly as the hollow nanodisk moves away from the optical axis. When the hollow nanodisk locates at the position of $x=105 \mathrm{~nm}$, the LRR of $8 \mathrm{~dB}$ is obtained. For other wavelengths, the dependence of the scattering directivity on the lateral displacement is similar. For the case of shorter wavelengths, there is a bit difference between the results obtained by the FDTD method and the theoretical model only including the overlapping of TED and MD, which can be attributed to the influence of higher-order moments. However, it should be noted that the linear relationship of the scattering directivity with the lateral displacement still almost remains. Therefore, the results in Fig. $5(\mathrm{c})$ indicate that in terms of the variation of the position-dependent directivity, transverse displacement sensing can be achieved within a broadband wavelength range in the near-infrared region.

When the hollow nanodisk moves away from the focal plane along the $z$ direction, both of the amplitudes of the longitudinal electric-field component and transverse magnetic-field component decrease $\frac{[33]}{}$. There is nearly no variation in the relative strength of $\left|m_{y} / c D_{z}\right|$ with increasing $z$ displacement, and $z$ displacement sensing could not be realized. However, due to the different tendencies of the longitudinal electric-field component and 
transverse magnetic-field component along the transverse direction $-[21,23,33]$, transverse displacement sensing is still valid when the hollow nanodisk does not locate at the focal plane.

In summary, we propose a scheme to realize broadband transverse displacement sensing based on the interaction of a focused RP beam with a silicon hollow nanodisk. Using the multipolar decomposition analysis, it is found that the interference of a longitudinal TED and a lateral MD is dominant in far-field scattering. The TED is almost in phase with the MD within a broad wavelength range, and the relative amplitude of those two dipoles changes as the hollow nanodisk moves away from the optical axis. As a result, broadband position sensing based on the sensitivity of scattering directivity to transverse displacement can be achieved, and the working wavelength range is enlarged as high as $155 \mathrm{~nm}$. The results show that hollow nanodisks can be used as versatile antennas for broadband position sensing and find potential applications, such as super-resolution microscopy, modern nanometrology, and integrated optical circuits.

This work was supported by the Key Program of the Natural Science Foundation of Tianjin (No. 19JCZDJC32700) and the Science and Technology Support Program of Tianjin (No. 17YFZCSY00740). This work was partially carried out at Engineering Research Center of Thin Film Photo-electronics Technology, Ministry of Education.

\section{References}

1. L. Novotny and N. van Hulst, Nat. Photon. 5, 83 (2011).

2. M. Alavirad, A. Olivieri, L. Roy, and P. Berini, Chin. Opt. Lett. 16, 050007 (2018).

3. I. Koirala1, C.-S. Park, S.-S. Lee, and D.-Y. Choi, Chin. Opt. Lett. 17, 082301 (2019).

4. D. Dregely, K. Lindfors, M. Lippitz, N. Engheta, M. Totzeck, and H. Giessen, Nat. Commun. 5, 4354 (2014).

5. T. Coenen, F. B. Arango, A. F. Koenderink, and A. Polman, Nat. Commun. 5, 3250 (2014).

6. M. Neugebauer, T. Bauer, P. Banzer, and G. Leuchs, Nano Lett. 14, 2546 (2014).

7. T. Shegai, S. Chen, V. D. Miljković, G. Zengin, P. Johansson, and M. Käll, Nat. Commun. 2, 481 (2011).

8. Y. H. Fu, A. I. Kuznetsov, A. E. Miroshnichenko, Y. F. Yu, and B. Luk'yanchuk, Nat. Commun. 4, 1527 (2013).

9. K. Yao and Y. Liu, ACS Photon. 3, 953 (2016).

10. J. M. Geffrin, B. García-Cámara, R. Gómez-Medina, P. Albella, L. S. Froufe-Pérez, C. Eyraud, A. Litman, R. Vaillon, F. González, M. Nieto-Vesperinas, J. J. Sáenz, and F. Moreno, Nat. Commun. 3, 1171 (2012).
11. A. Devilez, B. Stout, and N. Bonod, ACS Nano 4, 3390 (2010).

12. B. S. Luk'yanchuk, N. V. Voshchinnikov, R. Paniagua-Domínguez, and A. I. Kuznetsov, ACS Photon. 2, 993 (2015).

13. S. Zhang, R. Jiang, Y.-M. Xie, Q. Ruan, B. Yang, J. Wang, and H.-Q. Lin, Adv. Mater. 27, 7432 (2015).

14. I. Staude, A. E. Miroshnichenko, M. Decker, N. T. Fofang, S. Liu, E. Gonzales, J. Dominguez, T. S. Luk, D. N. Neshev, I. Brener, and Y. Kivshar, ACS Nano 7, 7824 (2013).

15. T. Shibanuma, T. Matsui, T. Roschuk, J. Wojcik, P. Mascher, P. Albella, and S. A. Maier, ACS Photon. 4, 489 (2017).

16. J. Li, N. Verellen, D. Vercruysse, T. Bearda, L. Lagae, and P. Van Dorpe, Nano Lett. 16, 4396 (2016).

17. H.-S. Ee, J.-H. Kang, M. L. Brongersma, and M.-K. Seo, Nano Lett. 15, 1759 (2015).

18. Y. Wang, X. Zeng, E. Yang, Y. Lu, D. Zhang, and P. Wang, Chin. Opt. Lett. 14, 011601 (2016).

19. T. Das, P. P. Iyer, R. A. DeCrescent, and J. A. Schuller, Phys. Rev. B 92, 241110 (2015).

20. Z. Xi, L. Wei, A. J. L. Adam, and H. P. Urbach, Opt. Lett. 41, 33 (2016).

21. S. Nechayev, J. S. Eismann, M. Neugebauer, P. Woźniak, A. Bag, G. Leuchs, and P. Banzer, Phys. Rev. A 99, 041801 (2019).

22. M. Neugebauer, P. Woźniak, A. Bag, G. Leuchs, and P. Banzer, Nat. Commun. 7, 11286 (2016).

23. A. Bag, M. Neugebauer, P. Wozniak, G. Leuchs, and P. Banzer, Phys. Rev. Lett. 121, 193902 (2018).

24. F. Deng, H. Liu, M. Panmai, and S. Lan, Opt. Express 26, 20051 (2018).

25. W. Shang, F. Xiao, W. Zhu, H. Lei, M. Premaratne, T. Mei, and J. Zhao, Opt. Express 27, 4944 (2019).

26. Y. Wang, Y. Lu, and P. Wang, Opt. Express 26, 1000 (2018).

27. N. Tischler, J. Stark, X. Zambrana-Puyalto, I. Fernandez-Corbaton, X. Vidal, G. Molina-Terriza, and M. L. Juan, ACS Photon. 5, 3628 (2018).

28. Z. Xi, L. Wei, A. J. L. Adam, and H. P. Urbach, Phys. Rev. Lett. 117, 113903 (2016).

29. M. X. Zhang, Q. Zhang, S. J. Zeng, Z. Z. Liu, and J.-J. Xiao, Opt. Lett. 43, 1275 (2018).

30. J. Lv, H. Mu, Q. Liu, X. Zhang, X. Li, C. Liu, S. Jiang, T. Sun, and P. K. Chu, Appl. Opt. 57, 4771 (2018).

31. T. Feng, W. Zhang, Z. Liang, and Y. Xu, J. Phys: Condens. Matter 32, 124002 (2018).

32. E. D. Palik, Handbook of Optical Constants of Solids (Academic, 1991).

33. K. S. Youngworth and T. G. Brown, Opt. Express 7, 77 (2000).

34. A. B. Evlyukhin, T. Fischer, C. Reinhardt, and B. N. Chichkov, Phys. Rev. B 94, 205434 (2016).

35. A. E. Miroshnichenko, A. B. Evlyukhin, Y. F. Yu, R. M. Bakker, A. Chipouline, A. I. Kuznetsov, B. Luk'yanchuk, B. N. Chichkov, and Y. S. Kivshar, Nat. Commun. 6, 8069 (2015).

36. P. D. Terekhov, K. V. Baryshnikova, Y. A. Artemyev, A. Karabchevsky, A. S. Shalin, and A. B. Evlyukhin, Phys. Rev. B 96, 035443 (2017). 